\title{
The optimum choice of gate width for neutron coincidence counting
}

\author{
S. Croft ${ }^{1 *}$, D. Henzlova ${ }^{2}$, A. Favalli ${ }^{2}$, D. Hauck ${ }^{2}$, and P.A. Santi ${ }^{2}$ \\ ${ }^{1}$ Safeguards \& Security Technology (SST), Global Nuclear security Technology \\ Divisions, PO Box 2008, Bldg 5700, MS-6166,Oak Ridge, TN 37831-6166, USA \\ ${ }^{2}$ Safeguards Science \& Technology Group (NEN-1), Nuclear Engineering and \\ Nonproliferation Division, MS-E540, Los Alamos, NM 87545, USA \\ *Corresponding author. Email crofts@ornl.gov. Phone +1 (865)-241-2834
}

\begin{abstract}
In the measurement field of international nuclear safeguards, passive neutron coincidence counting is used to quantify the spontaneous fission rate of certain special nuclear materials. The shift register autocorrelation analysis method is the most commonly used approach. However, the Feynman-Y technique, which is more commonly applied in reactor noise analysis, provides an alternative means to extract the correlation information from a pulse train. In this work we consider how to select the optimum gate width for each of these two time-correlation analysis techniques. The optimum is considered to be that which gives the lowest fractional precision on the net doublets rate. Our theoretical approach is approximate but is instructional in terms of revealing the key functional dependence. We show that in both cases the same performance figure of merit applies so that common design criteria apply to the neutron detector head. Our prediction is that near optimal results, suitable for most practical applications, can be obtained from both techniques using a common gate width setting. The estimated precision is also comparable in the two cases. The theoretical expressions are tested experimentally using ${ }^{252} \mathrm{Cf}$ spontaneous fission sources measured in two thermal well counters representative of the type in common use by international inspectorates. Fast accidental sampling was the favored method of acquiring the Feynman-Y data. Our experimental study confirmed the basic functional dependences predicted although experimental results when available are preferred. With an appropriate gate setting Feynman-Y analysis provides an alternative to shift register analysis for safeguards applications which opening up new avenues of data collection and data reduction to explore.
\end{abstract}

Keywords: neutron coincidence counting; point-model; shift register; Feynman-Y 


\section{Introduction}

Ensslin [1] provides an approximate plausibility argument, which has been shown to hold reasonably well in practice $[2,3]$, that closey predicts the optimum gate width for use in shift register based coincidence counting. When the accidentals (or chance) coincidence rate far exceeds the real (or genuine) coincidence rate the gate width that returns the lowest relative standard deviation on the net real rate is predicted to be about 1.26 times the nominal 1/e neutron dieaway time of the detector system.

In this work we review Ensslin's derivation and adopt the same general reasoning to answer the question of what the optimum gate width is when Feynman-Y analysis [4] is being used to extract the correlation information from the pulse train rather than shift register logic. We don't expect the estimate to be completely accurate, because the pulse train from fission sources, by definition, is correlated and our theory does not account fully for this, but we do expect it to reveal the key functional dependences according to the current traditional understanding.

Traditionally multiplicity shift register (MSR) analysis records a multiplicity histogram to extract higher order moments (such as triplets) from the measurement multiplicity distribution. In recording the data, the MSR technique uses a single value of coincidence gate width that is chosen based on system characterization measurements. This is because traditional hardware devices usually only support a single gate width setting, although in future designs this limitation could well be lifted. In practice using a single gate width has been acceptable, even for general purpose applications, because the 
optimum setting, judged on the basis of minimizing the relative standard deviation on the net reals (coincidence or doubles) rate, for a given item lies at the bottom of a broad minimum provided the accidentals rate is dominant. In other words the exact choice of gate width under these conditions is usually not critical in the context of other sources of measurement uncertainty. Furthermore, for the small-mass items of safeguards interest, far from criticality, the neutron dieaway profile is utterly dominated by the characteristics of the neutron detector, not the assay item, and so the position of the minimum is not strongly dependent on the item either. For the assay of plutonium scrap and waste the situation is a little different in that the dieaway profile can be influenced by the matrix material and it may be prudent to use a wider gate (perhaps twice the dieaway time) to reduce any sensitivity to this effect. But in this case the usual analysis which assumes that the neutron detection efficiency is known and fixed is no longer valid either. To resolve this dependence a small ${ }^{252} \mathrm{Cf}$ source is introduced near the container and the matrix perturbation is determined directly. This so called add-a-source (AAS) or matrixinterrogation-source (MIS) approach therefore compensates for the change in the value of both the efficiency and the gate utilization factor (the fraction of correlated events on the pulse train that fall within the finite duration of the coincidence gate). Since the value of the gate width stays within the broad minimum the impact of not precisely choosing the optimal gate width is small. The exception to this shallow optimum rule of thumb is when assaying in the low-mass, low rate, regime, particularly close to the minimum detection limit, with chemically clean (low $(\alpha, n)$-to-spontaneous fission neutron ratio) materials (as opposed to plutonium-bearing materials which may have low mass but high contribution of $(\alpha, n)$ neutrons). In this extreme the accidentals rate becomes negligible 
and no longer strongly influence the precision on the net correlated rate calculated and so to achieve the 'best' counting statistics in reals it becomes more important to obtain the highest possible signal. In this counting regime, instead of a gate width of about 1.26 times the dieaway time (as detailed below) a value of 2 or 2.5 times the dieaway time might be used instead so as to essentially integrate over the region of correlation in the 1dimensional Rossi- $\alpha$ distribution. This choice then comes with the added benefit that variations in the gate utilization factor introduced by the matrix are also lessened (as was discussed for waste and scrap).

The optimum value of the predelay, $T_{p}$, is simply the minimum value necessary for the system to return to quiescent conditions following a detected event so that the accidentals subtraction might be made with negligible bias. The predelay is usually many times larger than the effective system dead time although it may be comparable to the maximum dead time of an individual counting channel. The predelay allows any baseline shift, which affects the trigger probability (efficiency) of small amplitude signals to dissipate. The predelay may be reduced by careful hardware design. But for a given system the predelay setting is not amenable to the statistical optimization in the way that the coincidence gate width is. Consequently we shall not discuss the choice of predelay further. 


\section{Optimum gate width selection - shift register (MSR) analysis}

In the conventional multiplicity shift register (MSR) approach to neutron coincidence counting [1] every neutron event (signal) recorded on the pulse train triggers the inspection of a coincidence gate of width $T_{g}$ after a short predelay, of duration $T_{p}$. The accumulated number of counts in the gate represents the number of pairs in coincidence with the triggering event. The count in the gate includes both genuine (or real) coincidences together with accidental (or chance) coincidences. For this reason this gate is called the 'reals plus accidentals' gate $(\mathrm{R}+\mathrm{A})$. To evaluate the accidental coincidence contribution, another gate is opened a long time, $T_{L}$, after the $(\mathrm{R}+\mathrm{A})$-gate is closed. The accumulated value of the number of counts in this gate gives the average number of accidental coincidences provided the long delay $T_{L}$ is many times longer than the effective 1/e dieaway time, $\tau$. Any genuine time correlation to the triggering event will then have dissipated after $T_{L}$ has elapsed. It is because of this that the late gate is called the accidentals or A-gate. If the total (gross or singles) counting rate is denoted by $S$, then, on average, the A-gate will be opened $S$ times every second and the average number of counts within each gate will be given by the product of the total event rate and the duration of the gate, $S \cdot T_{g}$. Thus under steady conditions the accidentals rate is expected to be given by $A=S^{2} T_{g}$.

To extract the genuine coincident rate, the information from $(\mathrm{R}+\mathrm{A})$-gate and A-gate is combined in the traditional MSR analysis. Working in terms of count like quantities (as opposed to rates) and treating the correlated signal as if it were the result of a matched 
pair of independent Poisson variates we have for the net reals rate and the associated standard deviation:

$R=\frac{1}{t}[(R+A) \cdot t-A \cdot t] \pm \frac{1}{t} \cdot \sqrt{[(R+A)+A] \cdot t}$

We emphasize that $\mathrm{R}$ and $\mathrm{A}$ are rates, so that $R \cdot t$ and $(R+A) \cdot t$ are counts, and we have made the assumption that the variance on the number of counts for a counting experiment is equal to the number of counts.

The reciprocal relative standard deviation in the limit $A \gg R$ then becomes:

$\frac{R}{\sigma_{R}} \sim \frac{R_{2} \cdot f_{2}}{\sqrt{2 . t . A}} \sim \frac{\sqrt{t}}{\sqrt{2}} \frac{1}{\sqrt{\tau}} \frac{R_{2}}{S} \frac{f_{2}}{\sqrt{T_{g} / \tau}}$

where $R_{2}$ is the second factorial moment rate, sometimes called the doublet or second order multiplet rate, with the meaning that it is the net reals rate for the item with perfect gating. The factor $f_{2}$ is the Gate Utilization Factor (GUF) [5] which accounts for the fact that not all coincidences present on the pulse train are counted because finite values of $T_{p}$ and $T_{g}$ are being used and some correlated events fall outside the gate. Perfect gating means the limiting result as $T_{p}$ approaches zero and $T_{g}$ becomes infinitely large compared to the lifetime of neutrons in the system for a detector free of instrumental artifacts (that is the reasons why we need a finite predelay are abscent).

For a given item one could plot the $\left(R / \sigma_{R}\right)$-ratio as a function of $T_{g}$ to find the value that gives the maximum. But here we want generic guidance based on theory rather detailed item specific information which will depend on the exact rates and temporal character of the system. For a detection system with a pure single exponential dieaway profile we 
have, upon substituting the theoretical expression for the GUF, $f_{2}$, and the calculated result for the accidentals rate $A=S^{2} \cdot T_{g}$ :

$\frac{R}{\sigma_{R}} \sim \frac{e^{-T p / \tau}}{\sqrt{2}} \cdot \sqrt{t} \cdot\left(\frac{1}{\sqrt{\tau}} \frac{R_{2}}{S}\right) \cdot \frac{\left(1-e^{-T_{g} / \tau}\right)}{\sqrt{T_{g} / \tau}}$

The maximization problem therefore reduces to finding the turning point of the function:

$\frac{\left(1-e^{-T g / \tau}\right)}{\sqrt{T_{g} / \tau}}=\frac{\left(1-e^{-z}\right)}{\sqrt{z}}=y(z)$

where $z=T_{g} / \tau$. In the limit $z \rightarrow 0$ we find $y(z) \rightarrow \sqrt{z} \rightarrow 0$ while in the limit $z \rightarrow \infty$ we find $y(z) \rightarrow 1 / \sqrt{z} \rightarrow 0$ and the two extremes are connected by a simple continuous curve possessing a single maximum. Numerical evaluation gives the position of the maximum as $z=T_{g} / \tau \sim 1.2564$ [see Note to analytical discussion], and the value of $y(z)$ at the peak $\sim 0.6382$. This is the justification for why the 'best' choice of gate width quoted earlier takes on the value it does in terms of the 1/e dieaway time for an ideal detector with a pure exponential dieaway profile. We note that it has been shown that this simple result is not strongly perturbed by non-Poisson behavior or dead time losses in real systems $[2,3]$. Because the extension to the theory to account for correlations does not alter main functional dependences we have elected to use the simpler treatment here which is also by far the most commonly encountered form.

Once $y(z)$ has been optimized the expression for $R / \sigma_{R}$ predicts the usual square root improvement as a function of counting time but is otherwise fixed by system design. Since, according to the point-model, the ratio $R_{2} / S$ is directly proportional to the counting efficiency, $\varepsilon$, we see that under the stated assumptions a good figure of merit for 
comparing the coincidence precision performance between different systems is the ratio $\varepsilon / \sqrt{\tau}$ as given by Evans et al [6]. This ratio shows quantitatively the importance of a high efficiency and a short dieaway time. For most practical systems this approximation provides reasonable guidance because the dependence of the precision on the exact setting of the gate width is rather weak in the vicinity of the optimum choice and also because departures from exponential behavior are of secondary importance (real neutron well counter systems often have multiple rings of ${ }^{3} \mathrm{He}$ proportional counters typically need three decay components to describe them well comprising a short live component and long lived tail and a dominant mode in between). An effective value works well since it describes the main dieaway sequence responsible for most of the observed signal.

\section{Optimum gate width selection - Feynman-Y (RTI) analysis}

In the case of Feynman-Y counting [4], which makes use of the variance-to-mean (VTM) less than unity as an estimator of the correlated signal, sampling gates are normally opened periodically, which is to say randomly with respect to the incoming pulse train, and the histogram of the number of counts, $i \geq 0$, observed is compiled. For this reason we refer to this kind of pulse train sampling for time correlation analysis as using Randomly Triggered Inspection intervals (RTI). Depending on the sampling rate the gates can be either non-overlapping or overlapping [4]. Conventionally two quantities are calculated from the count distribution, the Feynman-Y statistic and also $Q$. The Feynman-Y statistic is defined by the excess VTM through the relation:

$$
Y=V T M-1=\frac{\sigma^{2}}{\mu}-1=\frac{\left\langle i^{2}\right\rangle-\langle i\rangle^{2}}{\langle i\rangle}-1
$$


where $\sigma^{2}$ and $\mu$ denote the variance and mean of the count distribution respectively and we are using the $\langle\cdot\rangle$ notation to denote the average value of the enclosed quantity formed over the normalized observed experimental frequency distribution. For a perfectly random (Poissonian) pulse train the average value of the Feynman-Y statistic will be zero. When correlated events are present it will be non-zero in a way that depends on both the leakage self-multiplication of the item and the ratio $\alpha$ of the $(\alpha, n)$-to-spontaneous fission neutron production. The derivation of the relationship may be found in [4].

Q is defined as follows:

$Q=\sigma^{2}-\mu=\mu Y=\left\langle i^{2}\right\rangle-\langle i\rangle^{2}-\langle i\rangle=\langle i(i-1)\rangle-\langle i\rangle^{2}$

In terms of the RTI number distribution we can write the second order multiplet rate as follows [7,8]:

$R_{2}=\frac{1}{w_{2}} \cdot \frac{1}{2} \cdot \frac{\left[\langle i(i-1)\rangle-\langle i\rangle^{2}\right]}{T_{g}}=\frac{1}{w_{2}} \cdot \frac{1}{2} \cdot \frac{Q}{T_{g}}$

In this expression $w_{2}$ is the RTI GUF for pairs counting and for a system with a pure exponential dieaway profile is given by $[5,9]$ :

$w_{2}=1-\frac{\left(1-e^{-T_{g} / \tau}\right)}{T_{g} / \tau}$

We observe that $Q$ is directly proportional to the correlated second factorial multiplet rate $R_{2}$ because the accidental chance coincidences have been subtracted. Thus, the relative standard deviation due to counting statistics on our sought after quantity $R_{2}$ will be equal to the relative standard deviation in $Q$. 
To proceed in our estimation for the relative standard deviation suppose, as previously, that we have a high total rate but only weakly correlated pulse train, $R_{2} / S \ll 1$. Rewriting our expression for $R_{2}$ in terms of count-like quantities we have for the average quantities:

$2 w_{2} R_{2} t=\frac{Q}{T_{g}} t=\left[\frac{\langle i(i-1)\rangle}{T_{g}} t-S^{2} T_{g} t\right]=\left[2 w_{2} R_{2} t+A t\right]-A t$

where we have made use of the relation $S=\langle i\rangle / T_{g}$ and again written $A=S^{2} \cdot T_{g}$ for convenience.

Let us assume, equivalently as we did in the shift register case, that the difference on the right hand side of this equation can be approximately treated as the difference between two large and independent numbers of counts. It is expected that his will give us a functional form which will also yield reasonable numerical estimates provided the correlations on the pulse train are modest so that the modification to Poisson behavior is of secondary importance [3]. This is because we shall use the familiar propagation of variance formula which assumes underlying Poisson behavior in the number of counts. Thus, the reciprocal relative standard deviation in $R_{2}$ under the assumption $2 \cdot w_{2} \cdot R_{2} \ll$ $A$ can be written as follows:

$\frac{R}{\sigma_{R}} \sim \frac{2 \cdot w_{2} \cdot\left(R_{2} \cdot t\right)}{\sqrt{2 \cdot A \cdot t}}=\sqrt{2} \cdot \sqrt{t} \cdot\left(\frac{1}{\sqrt{\tau}} \frac{R_{2}}{S}\right) \cdot \frac{w_{2}}{\sqrt{T_{g} / \tau}}$

This has strong similarities with the earlier result for the case of shift register analysis but we see that for the case of RTI analysis the function to maximize is instead: 
$\frac{w_{2}}{\sqrt{T_{g} / \tau}}=\frac{\left(1-\frac{\left(1-e^{-z}\right)}{z}\right)}{\sqrt{z}}=y(z)$

where again $z=T_{g} / \tau$. In the limit $z \rightarrow 0$ we find $y(z) \rightarrow \sqrt{z} / 2 \rightarrow 0$ while in the limit $z \rightarrow \infty$ we find $y(z) \rightarrow 1 / \sqrt{z} \rightarrow 0$. The extremes are connected by a simple continuous curve possessing a single maximum. The numerical solution in this case gives the position of the maximum at $z=T_{g} / \tau \sim 2.1491$ [see Note to analytical discussion] and the value of $y(z)$ at the peak is $\sim 0.4017$.

\{Here Fig.1\} 


\section{Discussion}

According to these rudimentary arguments the assay for doublets (also commonly referred to as pairs, reals or doubles) in the MSR approach would be optimized in the $\operatorname{limit} A \gg R$ by a gate of about 1.26. $\tau$ whereas the RTI result for doublets would be best using a gate of about 2.15. $\tau$. Fortunately for both functions the fractional variation is rather modest about the position of the peak for quite large changes in $z$, but the RTI setting being the more forgiving of the two, and so with $z=T_{g} / \tau \sim 1.621$ both functions are only $\sim 1.26 \%$ worse than optimum. More generally for MSR approach $z=T_{g} / \tau \sim 0.9-1.7$ is within $2 \%$ of the optimum while for the RTI approach counting of doublets $T_{g} / \tau \sim 1.5-3.0$ is within $2 \%$ of optimal performance.

Based on these observations a single common compromise of $z=T_{g} / \tau \sim 1.6$ would seem to be a reasonable compromise suitable for both shift register based passive neutron coincidence counting and Feynman-Y analysis for items for which accidentals dominates and which do not influence the dieaway profile.

Of course with list mode data acquisition [10] one is not limited to using a single gate width and one can make different choices in the subsequent off-line analysis for the two techniques if it makes a worthwhile difference. Having a multiplicity shift register with multiple gates working in synchrony offers a similar choice and also, as does list mode, allows the dieaway time to be measured for each item, which can provide additional qualitative and quantitative benefit to the assay in some cases (for instance when multiplying items are assayed under reflective conditions). 
In the case of MSR approach it is straightforward to show that the optimum gate width for coincidence counting is also the optimum choice at high rates for all order multiplets for the case of an exponential detector because the corresponding GUFs are given by $f_{n}=f_{2}^{n-1}$. In the case of RTI gating the form of the time correlation counting equations immediately suggests, if we follow the same basic reasoning, that for triplets counting the optimum gate would maximize $w_{3} / z$, where $z=T_{g} / \tau$ and $w_{3}=1-2 \frac{1-e^{-z}}{z}+\frac{1-e^{-2 z}}{2 z}[5]$. The optimum value of $z$ in this case is about 1.8926 [see Note to analytical] and the maximum is again rather broad. If $z$ is chosen to lie in the interval 1.5 to 2.4 then $y(z)$ is within $2 \%$ of the peak value. Thus, we find that our earlier suggestion of a single value of about $z \sim 1.6$ would likely also be quite acceptable for triples counting too according to the crude model presented. The result $f_{n}=f_{2}^{n-1}$ is of course strictly a theoretical one and we use it here only to obtain generic results for approximate guidance. Often real thermal well counters will have $f_{3}<f_{2}^{2}$.

It is instructive to compare the predicted functional relations for the inverse reciprocal relative standard deviations for the two modes of coincidence counting. To recap we found:

$\left[\frac{R}{\sigma_{R}}\right]_{S T I \_ \text {max }} \sim \frac{e^{-T_{p} / \tau}}{\sqrt{2}} \cdot \sqrt{t} \cdot\left(\frac{1}{\sqrt{\tau}} \cdot \frac{R_{2}}{S}\right) \cdot\left[\frac{\left(1-e^{-T g / \tau}\right)}{\sqrt{T_{g} / \tau}}\right]_{\max }$

and

$\left[\frac{R}{\sigma_{R}}\right]_{R T I \_\max } \sim \sqrt{2} \cdot \sqrt{t} \cdot\left(\frac{1}{\sqrt{\tau}} \cdot \frac{R_{2}}{S}\right) \cdot\left[\frac{w_{2}}{\sqrt{T_{g} / \tau}}\right]_{\max }$ 
We note that both expressions favor a high value of the ratio $\left(R_{2} / S\right) / \sqrt{\tau}$ which as previously mentioned is proportional to $\varepsilon / \sqrt{\tau}$ within the familiar point-model interpretive equations. Thus, both the conventional shift register and the Feynman-Y approaches to correlation counting favor a detector with a high efficiency and a low dieaway time in order to maximize and concentrate the correlated signal in the face of the accidentals contribution. This seems reasonable intuitively because one would expect the precision on the coincidence rate to be improved by a detector that recorded as many of the time correlated neutrons as possible from a common burst and concentrated them over a short period so that accidental coincidences were minimized. The important message is that the design criteria are the same for both approaches and turning this around a detector optimized for one approach should also be a good choice for the other. This means that ideas and methods developed under the respective traditions can be implemented without the need to fundamentally change the detection systems.

For a typical neutron coincidence counter constructed using ${ }^{3} \mathrm{He}$ filled proportional counters embedded in a high density polyethylene moderator $T_{p} / \tau \sim 0.1$ and we have shown that the optimum gate width is about 1.2564 times the dieaway time. Substituting these numerical values we obtain:

$\left[\frac{R}{\sigma_{R}}\right]_{S T I \_ \text {max }} \sim 0.41 \sqrt{t} \cdot\left(\frac{1}{\sqrt{\tau}} \cdot \frac{R_{2}}{S}\right)$

Similarly substituting the result that the optimum gate width for Feynman-Y analysis is about 2.1491 times the dieaway time we obtain:

$\left[\frac{R}{\sigma_{R}}\right]_{R T I \_ \text {max }} \sim 0.57 \sqrt{t} \cdot\left(\frac{1}{\sqrt{\tau}} \cdot \frac{R_{2}}{S}\right)$ 
From these considerations we find that both the shift register and Feynman- $Y$ approaches appear to offer similar performance although RTI gating would seem to have an inherent counting precision advantage by a factor of approximately 1.4 times (depending on the exact hardware implementation which sets the value of the predelay factor $e^{-T_{p} / \tau}$ ) and a similar result was demonstrated in [11]. We also point out that both leading multiplicative factors can be reduced by a factor of $\sqrt{2}$. This can be achieved, if in the accidentals subtraction step we can engineer for the uncertainty in the number of accidentals counts to be determined with a statistical uncertainty which is negligible compared to the number of reals plus accidentals. Under stable measurement conditions (for instance when neutron sources are not being moved in the assay room) this is achieved by using the calculated accidentals $\left(S^{2} \cdot T_{g} \cdot t\right)$ based off the singles rate, or by sampling the accidentals gate at a far higher rate than the incoming event rate (that is at a high fixed internal clock frequency). In the next section we test these predictions experimentally.

\section{Experimental Work}

To demonstrate and test the above theoretical considerations, measurements were performed using both an Epithermal Neutron Multiplicity Counter (ENMC) [12] and a second generation High Level Neutron Coincidence Counter (HLNCC) [13]. The former represents the present state-of-the-art in neutron well counter designs used for safeguards. It has a high efficiency and short dieaway time to allow for accurate measurements of higher order moments (beyond singles and doubles). It consists $121{ }^{3} \mathrm{He}$ filled proportional counters of $25.4 \mathrm{~mm}$ outer diameter each with a partial pressure of $10 \mathrm{bar}$ in 
${ }^{3} \mathrm{He}$ and arranged in four concentric rings inside a high-density polyethylene annulus. The HLNCC represents a commonly deployed standard design counting extensively used by the IAEA inspectorate for neutron coincidence counting for a variety of general purpose measurements and is fielded at numerous nuclear facilities. The HLNCC consists of $1825.4 \mathrm{~mm}$ outer diameter ${ }^{3} \mathrm{He}$ gas filled counters (4 bar in this case) arranged in a single ring. Both counters have reflective end-plugs and the measurements cavities are lined with cadmium to prevent thermal neutrons entering from the moderator and consequently, despite the complex geometrical arrangements, both designs can be represented to a good approximation by a single dieaway time system. Basic parameters of both counters are summarized in Table 1.

\section{\{Here Table 1\}}

Measurements were performed with a range of physically small ${ }^{252} \mathrm{Cf}$ sources to study the counting rate dependence of the optimum gate width for a matrix free cavity. Low, medium and high rate ${ }^{252} \mathrm{Cf}$ sources (in relation to typical rates observed for safeguarded plutonium items) were measured in each counter. Neutron emission rates of the measured sources are summarized in Table 2, column 2. Singles rates, $S$, measured in each counter are also quoted. Note that the measurements were performed on different dates, hence the measured $S$ rates quoted in Table 2 differ more than just by the detection efficiency of each counter.

\section{$\{$ Here Table 2\}}

Data were acquired in list mode (the detection time of every event is written to file for post data acquisition processing) and analyzed using standard shift register-type gating as 
well as RTI gating. Shift register type analysis (MSR analysis) was performed using the predelay $T_{p}$ quoted in Table 1. Doubles as well as triples (for ENMC only) rates relative standard deviations (RSD) were extracted for a range of gate width values as shown in Figure 2-4. To extract the RSD, the data were acquired in series of short time intervals (i.e. measurement time was divided into a series of shorter intervals) to evaluate the scatter in the data. RSD was then calculated using the following expression:

$R S D=\sqrt{\frac{\sum_{i=1}^{N}\left(x_{i}-\bar{x}\right)^{2}}{N \cdot(N-1)}}$

where $x_{i}$ refers to $D_{i}$ or $T_{i}$ rates measured in $i$-th time interval, $\bar{x}$ corresponds to mean value of measured rates $(\bar{D}$ or $\bar{T})$ over the total measurement time and $N$ is the number of time intervals within the total measurement time.

For MSR type analysis, in addition to long delay, $T_{L}$, needed to allow any correlations on the pulse train following the triggering event to dissipate in order to obtain the true accidental coincidence contribution in the A-gate, a method known as fast accidentals (FA) was also utilized to generate the A-gate histogram by sampling the gate at a fixed high-frequency (denoted as FA on in the Figures). The most improvement comes when the sampling frequency is far higher than the total counting rate. In hardware we usually use 20 or $50 \mathrm{MHz}$ which covers all of the safeguards items of interest. For the present measurements there is no difference in using either of these sampling rates. When analyzing list mode data off line the FA sampling rate is user selectable. For the present analysis we used $1 \mathrm{MHz}$ which satisfies the criterion of being far greater than the total 
neutron event rate for these items. The traditional A-gate acquisition utilizing long delay $T_{L}$ is thus labeled as FA off. It has been shown [7,11] that use of FA reduces the RSD on the experimental doubles rate by approximately factor of 1.4 for favorable high-rate cases. Similarly, as in case of MSR analysis, two gating options are explored for RTI analysis - overlapping gates, where each gate opens at a fixed high-frequency and consecutive (non-overlapping contiguous) gates, where each gate starts as soon as the previous one ends. Results summarized in the following figures demonstrate the differences in precision achievable with these different gating schemes.

\section{\{Here Figure 2\}}

\section{$\{$ Here Figure 3\}}

\section{\{Here Figure 4\}}

The trends in Figures 2 - 4 illustrate the relatively shallow minimum as a function of gate widths as discussed in previous sections. Also as predicted earlier, the RTI analysis is indeed less sensitive to variations of RSD with gate width exhibiting a broader minimum than MSR analysis. Comparison of Figures 2 and 3 illustrates that for the case of the multiplicity counter (ENMC) the optimum gate width for higher order multiplets (triples shown in Figure 3) agrees well with the optimum extracted using doubles rates. Thus a common design objective for both autocorrelation analysis methods is appropriate.

Trends observed in Figures 2 and 4 can be used to extract the optimum gate width corresponding to the lowest doubles RSD. The resulting values are summarized in Table 3. From Table 3 it can be seen that optimum gate width depends on the neutron emission rate of the source with wider gate widths being favored for low rate sources (Cf1) for 
both analyses and both counters. This is understandable, as discussed earlier, since sources with an especially low neutron emission rate will have a lower rate of accidental coincidences thus allowing the use of a wider gate width to improve the net counting statistics on the correlated signal.

Utilizing the theoretical optimum gate width expressions derived in this paper, the optimum gate width value for the ENMC would correspond to about $1.26 \tau=24 \mu \mathrm{s}$ and $2.15 \tau=41 \mu \mathrm{s}$ for the MSR and RTI analysis, respectively. For HLNCC the predicted optimum gate width would correspond to $54 \mu$ s and $93 \mu$ s for MSR and RTI analysis, respectively. Comparing these values with the experimentally extracted values in Table 3 it can be seen that in case of ENMC the predicted optimum gate width is slightly overestimated for medium and high rate sources ( $\mathrm{Cf} 2$ and $\mathrm{Cf} 3 \mathrm{~b}$ ) for both, MSR and RTI analysis. Considering the broad range of RSD minimum, the agreement is better for HLNCC and the predicted optimum gate width in this case falls within the range of values extracted in Table 3. Thus, the theory presented is demonstrated to be a useful guide although experimental determination of the best gate width to use is recommended so that the nature of the items to be measured and the measurement conditions can be factored into the choice.

\section{$\{$ Here Table 3$\}$}

From the trends in Figures 2-4 it can be concluded that in the case of shift register analysis the precision of FA sampling of the A-gate was always favored over sampling at the incoming event rate. A $1 \mathrm{MHz}$ clock rate (much higher than any of the measured singles rate) was used to perform FA sampling. The same sampling was used also to extract the RTI (overlapping) multiplicity rates and corresponding RSD values. The use 
of this sampling always results in better precision than the use of consecutive gates. These trends confirm findings discussed in detail in [11]. For a better overview, the minimum doubles RSD values corresponding to optimum gate ranges quoted in Table 3 are summarized in Table 4.

\section{$\{$ Here Table 4\}}

\section{Conclusions}

Correlated neutron coincidence counting in nuclear safeguards is commonly performed using shift register autocorrelation logic. Feynman-Y counting is an alternative approach commonly encountered in sub-critical assembly and reactor noise analysis. Basic questions such as whether one approach has an inherent advantage over the other, or if the fundamental design criteria for the detector head are different for the two approaches have not been addressed previously. In this work we used a simple statistical-counting model to show that the design criteria, expressed as a figure of merit are the same. Further we made predictions as to the relative performance under high counting rate conditions. These predictions were investigated experimentally.

In safeguards the shift register analysis is currently the dominant approach and a short gate width commensurate with the dieaway time is traditionally favored to get the best precision in a limited counting time as item throughput is an important consideration. The gate utilization factors (for doubles and triples counting) are treated as system parameters which can be determined separately. In sub-criticality experiments Feynman-

$\mathrm{Y}$ is more common and extrapolation to infinite gate width is often made to eliminate the 
gate utilization as a parameter. This is why in that field longer gates are common place. We have shown that the shift register gate factor converges faster and that may be an advantage in application. However, in the safeguards application space, where the gate utilization factors can be measured separately and treated subsequently as known values, it seems both shift register and Feynman-Y approaches could be applied at close to optimum precision using a common gate width setting although if list mode data is available separate setting can be used without penalty.

Our experimental study confirms the basic functional dependences predicted within this paper although we recommend that experimental results take precedence because they are free from the statistical-counting model assumptions. This work represents an extension of our earlier systematic study focusing on comparison of shift register and Feynman-Y approaches [11] for safeguards applications. We note that fast accidental sampling in the MSR case and the use of overlapping gates in the case of RTI approach is always favored from the perspective of counting precision compared to the alternative of sampling at the input rate and using consecutive gates respectively. Both the MSR with fast accidentals and Feynman (RTI) approaches using overlapping gates yield comparable precisions for the correlated rates observed for the Cf-sources measured in this work, although the RTI method with overlapping gates does seem to be slightly superior by this metric. A natural extension of the current work is to develop a more rigorous theoretical treatment and also to use Monte Carlo simulations in order to predict precision more accurately. 
The present work was limited to ${ }^{252} \mathrm{Cf}$ which has a somewhat harder multiplicity distribution than ${ }^{240} \mathrm{Pu}$, has no $(\alpha, n)$ contribution, and is none multiplying. It does not span the full nature of real-world $\mathrm{Pu}$ items of nuclear safeguards concern. There is decades of experience using shift register analysis for field measurements and it is important to extend the present exploration to this richer domain of items to additional establish practical confidence. Precision in the multiplet rates is one important metric and the next phase will also assess the alternative analysis option introduced here influence assay accuracy.

\section{Acknowledgement}

This work was sponsored by the U.S. Department of Energy (DOE), National Nuclear Security Administration (NNSA), Office of Nonproliferation Research and Development (NA-22).

[Note] Although we used direct numerical methods to find the locations of the maxima we note that the mathematical conditions based on setting the analytical derivatives of the various $y(z)$ expressions results in the following relations, which also served as both a check on correctness and consistency of the results reported.

For MSR analysis: $1-(1+2 z) e^{-z}=0$

For RTI doublets: $(3-z)-(3+2 z) e^{-z}=0$

For RTI triplets: $4 z(3-z)-8 z(2+z) e^{-z}+4 z(1+z) e^{-2 z}=0$ 


\section{References}

[1] N. Ensslin, Principles of neutron coincidence counting, Chapter 16 In: D Reilly, N. Ensslin, H. Smith, Jr., and S. Kreiner (Eds), Passive Nondestructive assay of nuclear materials, US Nuclear Regulatory Commission report NUREG/CR-5550 (March, 1991).

[2] N. Dytlewski, M.S. Krick, and N. Ensslin, Measurement variances in thermal neutron coincidence counting, Nucl. Instrum. and. Meths. in Phys. Res. A327(1993)469-479.

[3] S. Croft, M.T. Swinhoe, and V. Henzl, Á Priori Precision Estimation for Neutron Triples Counting, Proc. of the Second International Conference on Advancements in Nuclear Instrumentation, Measurement Methods and their Applications - ANIMMA, 6-9 June, 2011, the ICC, Ghent, Belgium.

[4] S. Croft, A. Favalli, D.K. Hauck, D. Henzlova, and P.A. Santi, Feynman variance-tomean in the context of passive neutron coincidence counting, Nucl. Instrum. and Meths. in Phys. Res A686 (2012)136-144.

[5] S. Croft, R.D. McElroy Jr., and S. Kane, Coincidence gate utilization factors for neutron correlation counters with up to three components in the die-away profile. Proc. of the $11^{\text {th }}$ International Conference on Environmental Remediation and radioactive Waste management, September 2-6, 2007, Bruges, Belgium. Paper ID 7173.

[6] L.G. Evans, D. Henzlova, H.O. Menlove, M.T. Swinhoe, S. Croft, J.B. Marlow, and R.D. McElroy Jr., Nuclear safeguards ${ }^{3} \mathrm{He}$ replacement requirements, Journal of Nuclear Materials Management XL No.3(Spring 2012)88-96.

[7] S. Croft, R.D. McElroy Jr., S. Philips, M.F. Villani, and L.G. Evans, Dead Time Behaviors in Passive Neutron Correlation Counting, Waste Management Symposia WM’07 February 25 - March 1 2007, Tucson, Arizona, USA. Paper 7258.

[8] S. Croft, D. Henzlova, and D.K. Hauck, Extraction of correlated count rates using various gate generation techniques: Part I Theory, Nucl. Instrum. and Meths in Phys Res. A691 (2012)152-158.

[9] D.M. Cifarelli and W. Hage, Models for a three-parameter analysis of neutron signal correlation measurements for fissile material assay, Nuclear Instruments and Methods in Physics Research A251 (1986)550-563.

[10] D. Henzlova, H. O. Menlove, M. T. Swinhoe, J. B. Marlow, I. P. Martinez, and C. D. Rael, Neutron Data Collection and Analysis Techniques Comparison for Safeguards, Los Alamos National Laboratory report LA-UR-10-06373, in: Proceedings of IAEA Safeguards Symposium 2010, IAEA-CN-184/178. 
[11] D. Henzlova, S. Croft, H.O. Menlove, and M.T. Swinhoe, Extraction of correlated count rates using various gate generation techniques: Part II Experiment, Nuclear Instruments and Methods in Physics Research A691 (2012)159-167.

[12] H. O. Menlove, C. D. Rael, K. E. Kroncke, and K. J. DeAguero, Manual for the Epithermal Neutron Multiplicity Detector (ENMC) for Measurement of Impure MOX and Plutonium Samples, Los Alamos National Laboratory report LA-14088 (2004).

[13] H. O. Menlove and J. E. Swansen, A high-performance neutron time correlation counter, Nuclear Technology 71 (1985) 497-505. 


\section{FIGURE CAPTIONS}

Figure 1. Plots of the $y(z)$ functions used in the selection of the optimal gate width for the MSR and RTI coincidence counting approaches respectively. These are normalized to unity at their respective peaks. This figure illustrates that that both functions have broad maxima and that in the vicinity of $z=1.6$ both functions are close to their peak values.

Figure 2: The relative standard deviation (RSD) for the doubles rates measured in ENMC as a function of gate width for MSR (left) and RTI (right) analysis, respectively. The lines shown are only to guide the eye.

Figure 3: The relative standard deviation (RSD) for the triples rates measured in the ENMC as a function of gate width for MSR (left) and RTI (right) analysis. The lines shown are to guide the eye.

Figure 4: The relative standard deviation (RSD) for the doubles rates measured in HLNCC as a function of gate width.

\section{TABLE CAPTIONS}

Table 1: Overview of the basic operating parameters of the ENMC and HLNCC.

Table 2: Overview of the ${ }^{252} \mathrm{Cf}$ sources measured in ENMC and HLNCC.

Table 3: Overview of optimum gate width values extracted from the experimentally determined doubles RSD as shown in Figures 2 and 4.

Table 4: Overview of the doubles RSD values corresponding to the optimum gate width values summarized in Table 3 . 


\section{FIGURE 1}

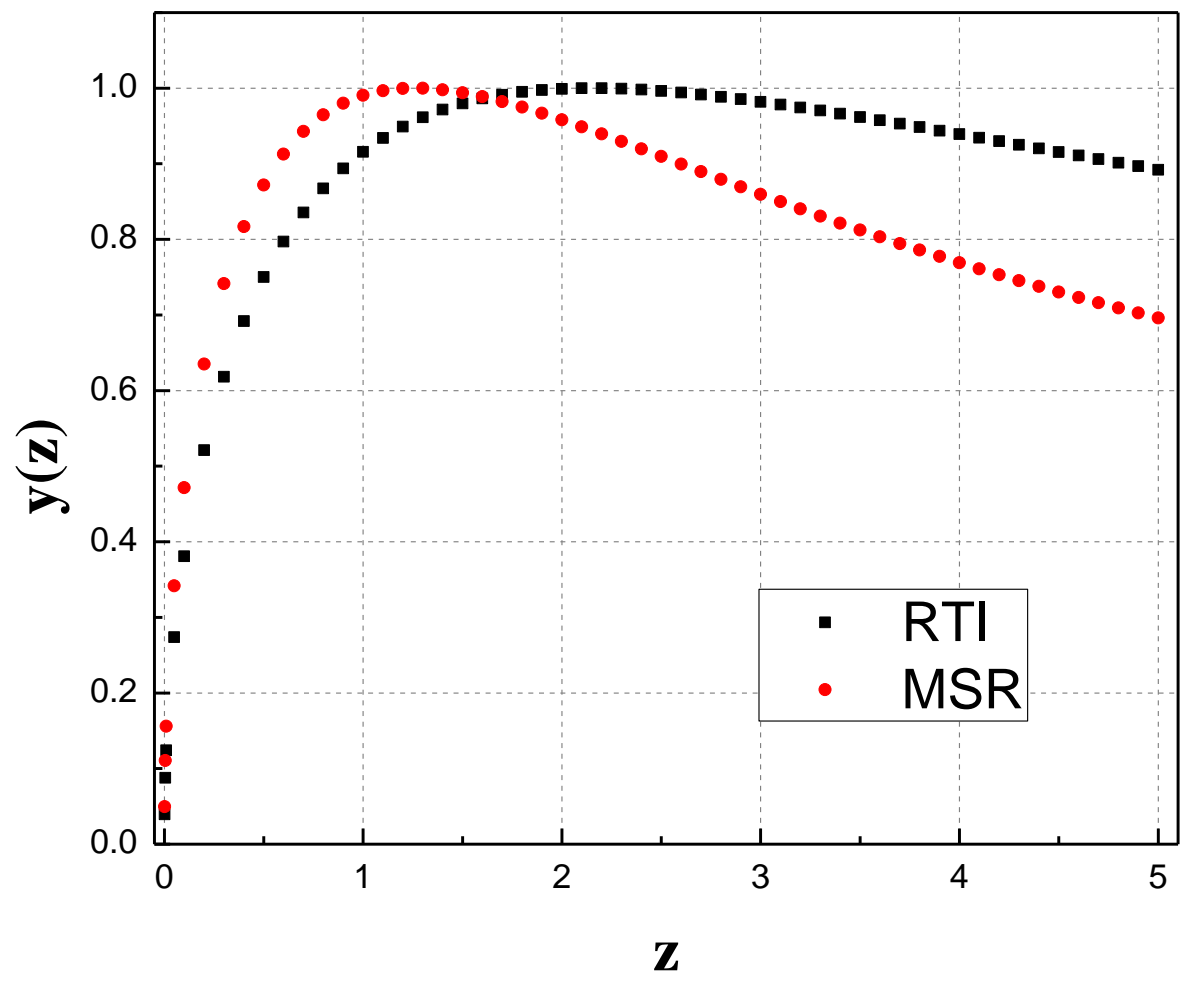




\section{FIGURE 2}
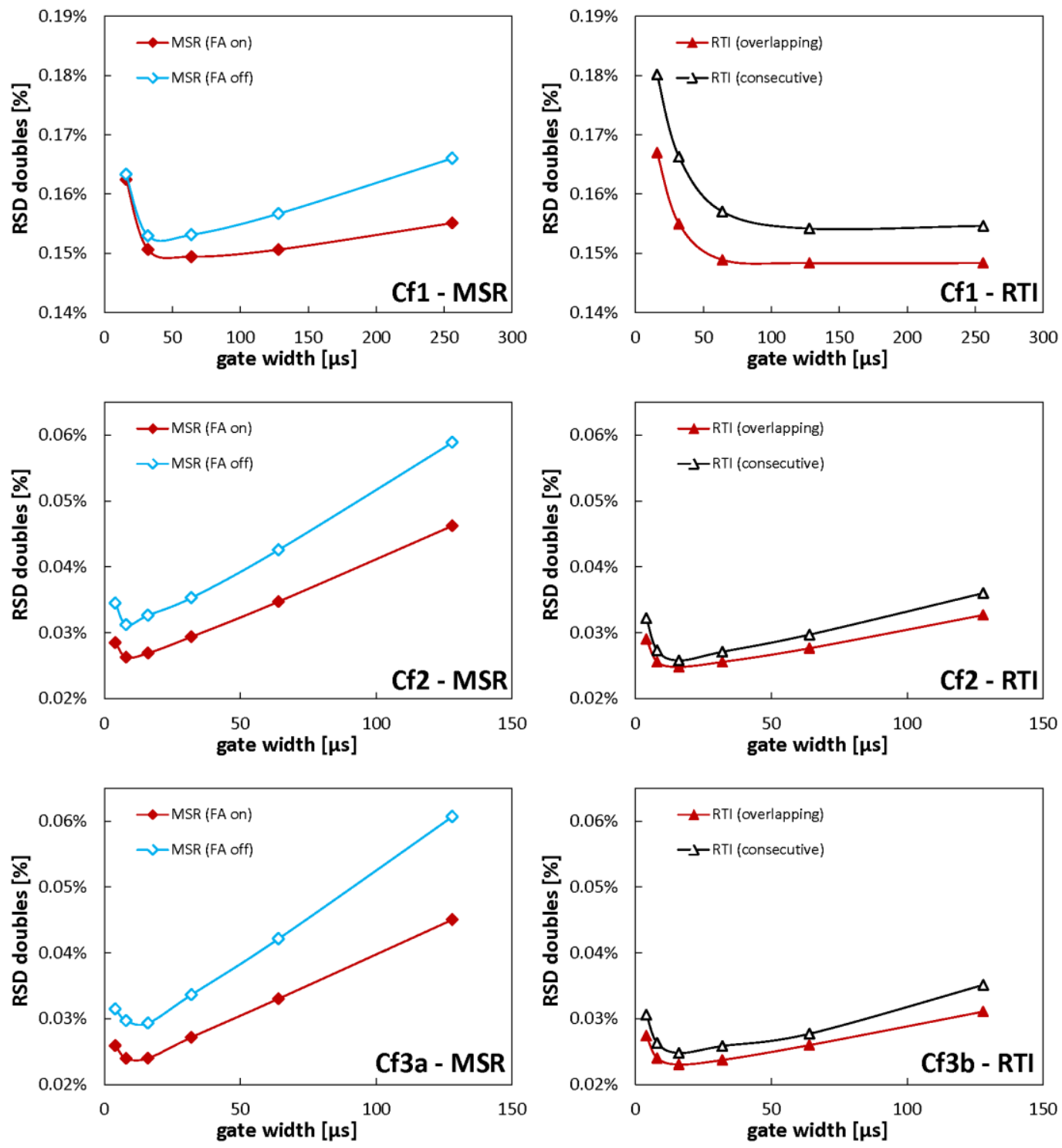


\section{FIGURE 3}
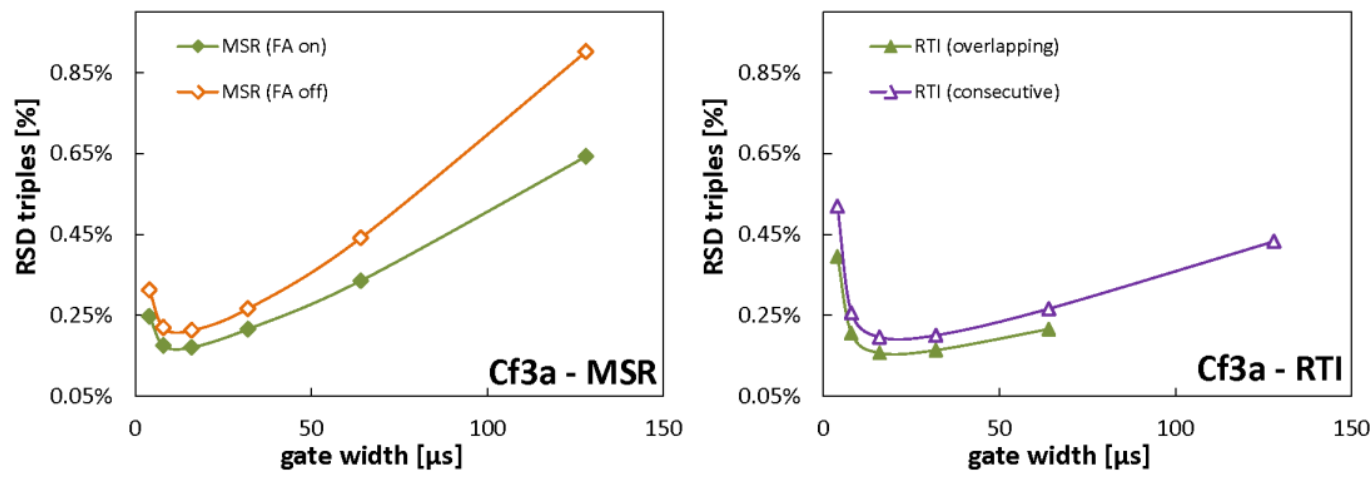


\section{FIGURE 4}
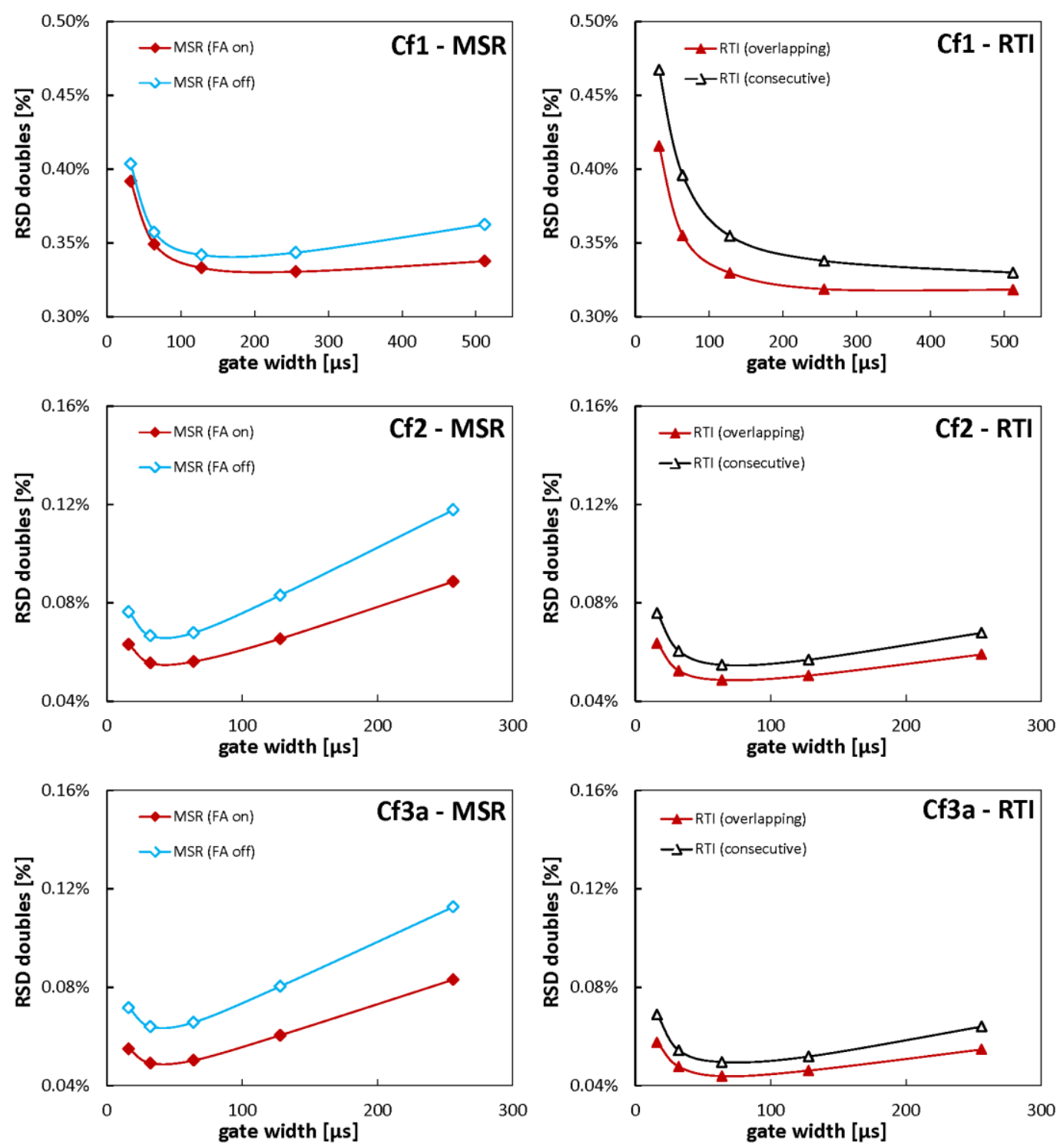
TABLE 1

\begin{tabular}{ccccc} 
Counter & $\begin{array}{c}\text { Predelay } \\
{[\mu \mathrm{s}]}\end{array}$ & $\begin{array}{c}\text { High Voltage } \\
{[\mathrm{V}]}\end{array}$ & $\begin{array}{c}\text { Dieaway } \\
{[\mu \mathrm{s}]}\end{array}$ & $\begin{array}{c}\text { Efficiency } \\
{[\%]}\end{array}$ \\
\hline ENMC & 1.5 & 1720 & 19.1 & 64.2 \\
HLNCC & 4.5 & 1680 & 43 & 17.1
\end{tabular}

TABLE 2

\begin{tabular}{cccccc} 
Source ID & $\begin{array}{c}\text { Source } \\
\text { strength } \\
{[\mathrm{n} / \mathrm{s}]^{*}}\end{array}$ & $\begin{array}{c}\text { S in ENMC } \\
{[\mathrm{cps}]}\end{array}$ & $\begin{array}{c}\text { S in } \\
\text { HLNCC } \\
{[\mathrm{cps}]}\end{array}$ & $\begin{array}{c}\text { Measurement } \\
\text { time ENMC } \\
{[\mathrm{h}]}\end{array}$ & $\begin{array}{c}\text { Measurement } \\
\text { time HLNCC } \\
{[\mathrm{h}]}\end{array}$ \\
\hline Cf1 & 667 & $357 \pm 0.4$ & $47 \pm 0.1$ & 2 & 4 \\
Cf2 & 131026 & $76937 \pm 6$ & $9299 \pm 1$ & 2 & 4 \\
Cf3a & 263265 & N/A & $18495 \pm 1$ & N/A & 4 \\
Cf3b & 508409 & $315783 \pm 12$ & N/A & 2 & N/A
\end{tabular}

* as of 01/01/2010. ENMC and HLNCC data were collected at widely separated times.

TABLE 3

\begin{tabular}{c|cc|cc}
\multirow{2}{*}{$\begin{array}{c}\text { Source } \\
\text { ID }\end{array}$} & $\begin{array}{c}\text { MSR } \\
\text { optimum GW } \\
{[\mu \mathrm{s}]}\end{array}$ & $\begin{array}{c}\text { RTI } \\
\text { optimum GW } \\
{[\mu \mathrm{s}]}\end{array}$ & $\begin{array}{c}\text { MSR } \\
\text { optimum GW } \\
{[\mu \mathrm{s}]}\end{array}$ & $\begin{array}{c}\text { RTI } \\
\text { optimum GW } \\
{[\mu \mathrm{s}]}\end{array}$ \\
\hline Cf1 & $32-64$ & $128-256$ & $128-256$ & $256-512$ \\
Cf2 & $8-16$ & $8-32$ & $32-64$ & $32-128$ \\
Cf3a & N/A & N/A & $32-64$ & $32-128$ \\
Cf3b & $8-16$ & $8-32$ & N/A & N/A
\end{tabular}

TABLE 4

\begin{tabular}{c|ccc|ccc} 
& \multicolumn{3}{|c|}{ ENMC RSD [\%] } & \multicolumn{3}{c}{ HLNCC RSD [\%] } \\
\hline Analysis method & Cf1 & Cf2 & Cf3b & Cf1 & Cf2 & Cf3a \\
\hline MSR (FA on) & 0.15 & 0.21 & 0.19 & 0.33 & 0.06 & 0.05 \\
MSR (FA off) & 0.15 & 0.26 & 0.24 & 0.34 & 0.07 & 0.06 \\
$\begin{array}{c}\text { RTI } \\
\text { (overlapping) }\end{array}$ & 0.15 & 0.20 & 0.18 & 0.32 & 0.05 & 0.05 \\
$\begin{array}{c}\text { RTI } \\
\text { (consecutive) }\end{array}$ & 0.15 & 0.21 & 0.20 & 0.33 & 0.06 & 0.06
\end{tabular}

